stained with haematoxylin and eosin, periodic acid Schiff's, Weil's myelin, Klüver's myelin, Nissl-cresyl violet, Bodian, and Congo red. On microscopic examination loss of neurons was most conspicuously present in layer 5 of the left precentral gyrus, most prominently of Betz cells. Neuronophagia (figure) and some proliferation of astrocytes was seen. The underlying white matter showed a decrease in the density of axons and slight gliosis. Other cortical areas, including the hippocampus, amygdala, and Broca's area showed no evident abnormalities. Although a single senile plaque was present, fibrillary tangles, Hirano bodies, Lewy bodies, amyloid depositions, and spongiform degeneration were not seen. The basal ganglia showed no abnormalities.

The clinical features of a right sided hemiparesis, slowly progressive in the course of three years, with only slight disturbances of intellect, in combination with postmortem evidence of non-specific loss of neurons in the motor cortex are indicative of local degenerative disease of the cerebral cortex. Alzheimer's and Pick's disease were ruled out on clinical and pathological grounds. In a series of six patients with progressive aphasia PET showed hypometabolism that was more extensive than the lesions visible on CT or $\mathrm{MRI},{ }^{1}$ as in our patient. The extensive involvement shown by SPECT was comparable with that in previously reported patients with isolated aphasia. $^{23}$

Our patient with an isolated hemiparesis of cortical origin, later accompanied by dysphasia, may represent a separate variety of a localised loss of cortical neurons, comparable with patients with isolated motor aphasia. ${ }^{1}$ The mild disturbances of intellectua functions on neuropsychological examination are largely explained by the hemiparesis and dysphasia. ${ }^{4}$ Mesulam has also pointed out that such disturbances may result from the influence of the original lesion on the function of other regions of the brain.

Patients with localised cortical atrophy in non-language areas and without dementia have been described in several groupsnamely, visuoperceptual disorder ${ }^{2}$; generalised apraxia; ${ }^{2}$ perceptuomotor deficits often combined with hemiparesis ${ }^{2}$ and behavioural and personality changes ${ }^{23}$; and progressive aphasia with unilateral extrapyramidal signs. ${ }^{5}$ The underlying pathological process in these localised cortical diseases is incompletely understood and not uniform. One patient with a slowly progressive aphasia showed (on biopsy) only non-specific and slight degenerative changes. ${ }^{1}$ One patient with a neuropsychiatric syndrome showed spongiform changes and mild gliosis in superficial layers of the prefrontal cortex. ${ }^{2}$ Three other patients with aphasia showed atrophy, spongiform changes, and astrocytosis, mainly in one or two layers of the cortex, without Pick or Lewy-type inclusion bodies.

It seems that primary progressive hemiparesis is comparable with primary aphasias or other focal degeneration syndromes of the cerebral cortex. This variety of disorders follows a more protracted course than the more generalised dementias, and has no morphological characteristics to link it with Alzheimer's or Pick's disease.

We thank M H Dijkman for referring the patient, Dr J M Keizer for information about the patient, Dr $P$ Maquet, for performing PET in 1988 in
Liège, Belgium, L M P Ramos for his interpretation of the radiological studies, and A F M M Verdonck for the neuropsychological examina-
tions.

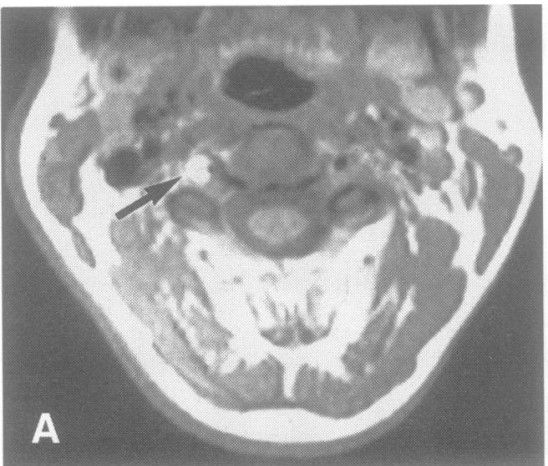

Mesulam MM. Primary progressive aphasiaNeurol 1987;22:533-4.

2 Caselli RJ, Jack CL. Asymmetric cortical degeneration syndromes. Arch Neurol 1992; 49:770-80.

3 Snowden JS, Neary D, Mann DMA, Goulding PJ, Testa HJ. Progressive language disorder due to lobar atrophy. Ann Neurol 1992;31:174-83.

4 Poeck K, Luzatti C. Slowly progressive aphasia in three patients, the problem of aphasia in three patients, the problem of accompanying neuropsych

5 Goulding PJ, Northing B, Snowden JS Progressive aphasia with right-sided extrapyramidal signs: another manifestation of localized cerebral atrophy. $\mathcal{f}$ Neurol Neurosurg Psychiatry 1989;52:128-9.

\section{Vertebral artery dissection mimicking} migraine

We agree with Giroud et al that internal carotid artery dissection is a major cause of cerebral infarction in those under 50 years of age. ${ }^{1}$ We also suggest that spontaneous dissection of the vertebral artery is an important cause of ischaemic stroke in this age group, increasingly recognised with the advent of effective, non-invasive methods of diagnosis such as colour duplex ultrasound and MRI. ${ }^{23}$ We report a patient presenting with symptoms that would not necessarily have justified investigation by conventional angiography but who was found, by noninvasive means, to have had a vertebral artery dissection.

A 35 year old woman, previously well, suddenly experienced distorted vision while doing the housework. She described wavy, flickering lines in both eyes and hazy vision that persisted for about an hour. As the flickering faded she became aware that she could only see the right half of her husband's face. This field defect persisted for about 30 minutes before her vision returned to normal. The next day she was aware of mild, diffuse headache and a stiff neck, which gradually improved over the next two weeks. There were no other associated symptoms and no history of trauma to the neck, although she had been playing in a competitive game of netball earlier in the day. In the past, she had had hypertension while pregnant but there was no history of prior cerebrovascular events or of migraine. She did not smoke.

Examination two weeks after the onset of symptoms was normal other than slightly hyperextensible little fingers on both hands. Cardiovascular examination was normal blood pressure was $130 / 80 \mathrm{~mm} \mathrm{Hg}$ and no bruits were audible in the neck. Skin and other joints were normal and there were no focal neurological signs.

It was considered that her symptoms could have been caused by a vertebrobasilar transient ischaemic episode. Migraine was also a possibility. Blood tests, including

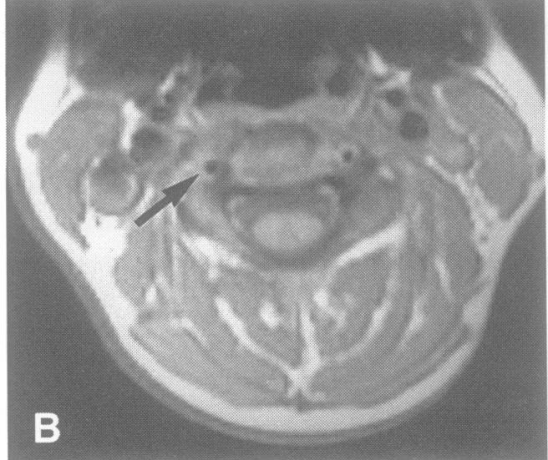

(A) Blood (white) in the wall of the right vertebral artery obstructing the lumen; $(B)$ the vertebral artery has now returned to normal.

blood count, glucose, cholesterol, lupus anticoagulant, anticardiolipin antibody concentrations, and syphilis serology were normal. Magnetic resonance imaging of the neck with axial, spin echo, $\mathrm{T} 1$ weighted images showed bright intramural thrombus with a very small residual lumen in the right vertebral artery (figure (A)). The abnormality was localised to a $2 \mathrm{~cm}$ portion of extracranial vertebral artery at the level of the C2 vertebra. Magnetic resonance angiography (MRA) showed disturbed flow within the vertebral artery at this level. The changes were diagnostic of vertebral artery dissection.

The patient was advised to continue aspirin for six months and has had no furafter the episode, showed no evidence of a persisting lesion (figure (B)).

Extracranial vertebral artery dissection, with occlusion or distal embolisation, is an important cause of transient ischaemic attack and stroke in patients under 50 years of age. Often there is no history of trauma to the neck or, when a history of trauma is present, it may be trivial. Epidemiological studies may have underestimated the true incidence of both carotid and vertebral artery dissection because of the requirement in the past for invasive angiography, with its attendant risk, to make the diagnosis. With the introduction of routinely available ultrasound and MR techniques, allowing non-invasive diagnosis, there is little doubt that more cases will come to light. It is interesting to note that the incidence of carotid dissection seems to be similar to that of aneurysmal subarachnoid haemorrhage.

This woman's symptoms were "trivial" diagnosed as an attack of atypical migraine with no further investigation performed. ther symptoms. Repeat MRI, four months and the episode could easily have been 
Plain MRI of the neck and head, together with ultrasound, are probably the investigations of choice; MRA may prove helpful

G YOUNG

The Walton Centre for Neurology Rice Lane, Liverpool L9 $1 A E$, UK

\section{Correspondence to: Dr P Humphrey.}

1 Giroud M, Fayolle H, Andre N, Dumas $R$ Becker F, Martin D, et al. Incidence of internal carotid artery dissection in the community of Dijon. [letter]. $\mathcal{F}$ Neurol Neurosur Psychiatry 1994;57:1443.

2 Sturzenegger M, Mattle HP, Rivoir A, Rihs F Schmid C. Ultrasound findings in spontaneous vertebral artery dissection. Stroke 1993;24:1910-21.

3 Hoffman M, Sacco RL, Chan S, Mohr JP Non-invasive detection of vertebral artery dissection. Stroke 1993;24:815-9.

\section{Headache in lateral medullary infarc-} tion

Headache is a frequent, although underemphasised, symptom of lateral medullary infarction. Fisher reported headache in 22 of 41 patients with lateral medullary infarction. ${ }^{1}$ This frequency (54\%) is much higher than that of cerebral infarction in general in large series (12 to $38 \%) .^{2-5} \mathrm{We}$ studied the incidence and features of headache in 34 patients with lateral medullary infarction.

These patients comprised 28 men and six women, mean age $\mathbf{5 4 . 4}$ (range 35 to 72 ) years. The diagnosis of lateral medullary infarction was confirmed by MRI for 30 of the patients. We interviewed all the patients regarding the presence, timing, localisation, side, and quality of their headaches. Angiography was performed on 14 of the patients.

Headache occurred in 26 patients (76\%) It began in association with the neurological deficit in 13 patients, preceded the onset of stroke by one to five days in 10 , and by 15 to 58 days in three. We limited further analysis to the 23 patients with headache in close temporal relation to the onset of the stroke. Nineteen of the 23 patients had lateralised headache on the side of the infarct, which was occipital or cervical in $15(65 \%)$, frontotemporal in two $(9 \%)$, diffuse in one $(4 \%)$, and affecting the eye to forehead in one $(4 \%)$. It was bioccipital in three patients $(13 \%)$ and generalised in two $(9 \%)$. Headache was throbbing in 13 patients $(57 \%)$, eight of whose headaches were timed to their heart beat. It was described as dull or pressing in two (9\%), intense in four $(17 \%)$, and pricking in one $(4 \%)$.

Proposed mechanisms of vascular occlusion were atherothrombosis in 22 patients, cardiogenic embolism in four, vasculitis of the vertebral artery associated with systemic lupus erythematosus in one, dissection of the vertebral artery in one, fibromuscular dysplasia of the vertebral artery in one, haemodynamic in one, and indeterminate in four. Headache was more common in association with atherothrombotic infarcts $(16 / 22 ; 73 \%)$; than with cardioembolic infarcts $(0 / 4 ; 0 \%)$. Of the 14 patients with angiographically confirmed lesions of the vertebral artery, including occlusion in eight patients (atherothrombotic six, dissection one, fibromuscular dysplasia one) and severe hypoplasia in-six, 13 experienced headache. Nine angiograms included full neck views and five did not.

Headache was not related to the coexistence of cerebellar infarction or to its size. Of the 10 patients with cerebellar infarction in the territory of the posterior inferior cerebellar artery, six had headaches. Twenty of the 24 patients with infarction confined to the lateral medulla complained of headache.

Ten patients had pain in the eye, nose, and cheek, and all experienced headaches. All but one could distinguish facial pain from headache by its nature (burning, sore, unbearable), location, and appearance at the onset of stroke.

Our study confirms that headache is much more frequent in lateral medullary infarction than in cerebral infarction in general, although these studies suggest that headache is more likely to occur in posterior circulation ischaemia. ${ }^{2-5}$ The underlying cause of headache in cerebral infarction is unknown. Various theories have been proposed $^{2-4}$-namely, dilatation of an artery by a thrombus or embolus, dilatation of collateral circulation, release of a local endogenous chemical agent from the platelets, displacement of pain sensitive cerebral structures, and tension headache caused by emotional stress.

Our suggestion that headache in lateral medullary infarction is related to thrombus formation in the vertebral artery may support the platelet hypothesis. Headache in lateral medullary infarction was likely to occur in patients who had such conditions that resulted in thrombus formation especially in the vertebral artery (atherothrombosis, severe hypoplasia, and fibromuscular dysplasia), whereas none of the four patients with cardiogenic embolism complained of headache. In about half of the patients, headache preceded the onset of stroke and was throbbing in nature. In our study, dissection of the vertebral artery, which is a common cause of stroke in young people, might be underestimated because only nine of the 34 patients had angiography that included full neck views. Possibly some thromboses were secondary to dissection, especially in younger patients.

The head pain that accompanies lateral medullary infarction is thought to have two components; one engendered by the process occurring in the artery, the other the result of lesion in the central trigeminal system. In Fisher's series of 28 patients with head pain, 12 experienced only headache, six only facial pain, and 10 both. In our study, 26 of the 34 patients had headaches, 10 of whom also had facial pain. Facial pain in lateral medullary infarction usually occurs at the onset of stroke, has a characteristic nature (burning, stinging, unbearable, soreness), and is followed by numbness. ${ }^{1}$ Most of our patients who had both facial pain and headache could differentiate between them when carefully questioned. The high incidence of headache in lateral medullary infarction is not due to contamination by facial pain.

We conclude that headache is a very frequent accompanying or prodromal symptom in patients with lateral medullary infarction and that late life onset, occipital, throbbing headache has clinical relevance as a warning sign of lateral medullary infarction especially in the atherothrombotic subgroup.

SATOSHI KUWABARA KEIZO HIRAYAMA Department of Neurology, Chiba University School of Medicine, 1-8-1 Inohana, Chuo-ku, Chiba 260, fapan

Correspondence to: Dr Kuwabara.
1 Fisher CM. Headache in cerebrovascular disease. In: Vinken PJ, Bruyn GW, eds. Handbook of clinical neurology. Headache and cranial neuralgias. Vol 5, Amsterdam: North Holland Publishing Co., 1968:132-4.

2 Edmeads J. The headache of ischemic cerebrovascular disease. Headache 1979;19: 345-9.

3 Portenoy RK, Abissi CJ, Lipton RB, et al. Headache in cerebrovascular disease. Stroke 1984;15:1009-12.

4 Koudstaal PJ, van Gijin J, Kappelle LJ. Headache in transient or permanent cerebral ischemia. Stroke 1991;22:754-9.

5 Vestergaard $K$, Andersen G, Nielsen MI, Jensen TS. Headache in stroke. Stroke 1993; 24:1621-4.

Supernumerary phantom limb after right hemispheric stroke

In a previous paper, ${ }^{1}$ we noted that there are few published cases of supernumerary phantoms after cerebral lesion. Yet whether this rarity reflects a genuinely low incidence of the phenomenon, a failure to inquire by the examiner, or an unwillingness on the part of patients to report a delusion that they themselves may regard as bizarre and distressing is unclear. ${ }^{2}$ The last issue raises the question of whether there is a coherent pattern in patients' descriptions of their experience (and a consistent neurological basis for the condition).

We have recently seen a second case of supernumerary phantom limb in whom the symptomatology was strikingly similar to our earlier case. ${ }^{1}$ The current patient was an 80 year old, right handed man who was a retired teacher of mathematics. Against a background of hypertension of many years standing, he presented with a right hemispheric stroke on 3 March 1994. Examination showed a dense left hemiparesis, left sensory loss, left homonymous hemianopia, and gross left neglect. His Barthel score (an index of functional disability in daily living) was $4 / 20$ (severely disabled). He ignored people on his left side, bumped into doors and furniture on the left and failed to make left turns when manoeuvring his wheelchair. Left neglect was also apparent when reading text; words on the left of the page were missed.

Computed tomography (6 April 1994) showed a very extensive area of low density in the distribution of the right middle cerebral artery. This is a mass effect with effacement of the right lateral ventricle and cortical sulci. There was no evidence of haemorrhage.

We examined the patient on 15 September 1994 (six months after his stroke) in the course of an investigation of anosognosia. The following extract is the pertinent part of the interview ( $P=$ patient; $\mathrm{E}=$ examiner):

P: I had a stroke.

E: How did the stroke affect you?

P: I don't drive and I don't do crosswords any more.

E: Anything else?

P: I carry a very heavy hand around with me.

E: How many hands do you have?

P: Would it surprise you if I said I had three?

E: Yes, it would. Do your hands work well?

P: My right hand is fine but the left arm is paralysed. The third one I carry it about and I sometimes place it here (points with right hand to right upper leg). It's concrete, but I can move the fingers. 